\title{
Análisis cuali-cuantitativo de menús infantiles ofrecidos en restoranes familiares y de comida rápida en Santiago de Chile
}

\author{
Q ualitative-quantitative analysis of \\ children's menus offered in family and \\ fast food restaurants in Santiago, Chile
}

\begin{abstract}
Childhood obesity in Chile is at its highest history level of food consumption away from home and it is becoming more frequent; in turn, infant food offer is being questioned in the world by its relationship to childhood obesity and its unhealthy characteristics. In this study 45 children's menus of family and fast food restaurants were evaluated, conducting a qualitative and quantitative analysis. The obtained results showed that most of the menus exceeded nutritional recommendations in fat and sodium, and $40 \%$ of them provided over 800 calories, considered half of energy recommendations for a child per day. In conclusion, the energy input supplied by the analyzed infant menus exceeds the requirements of children, there is no direct relationship between price and nutritional quality, and the children's menus have few healthy properties, $80 \%$ being classified as bad or very bad according to Chilean Dietary Guidelines 2013.
\end{abstract}

Key words: Childhood obesity, children's menus, fast food, family restaurants, food regulation.

\section{INTRODUCCIÓN}

Uno de los problemas más comunes asociado al estilo de vida actual es el exceso de peso. Este se presenta cuando una persona ingiere más energía de la que necesita generando un balance energético positivo, calificándose como sobrepeso (SP) y obesidad (OB) de acuerdo a su magnitud, entendiendo a ambos como un peso elevado para la talla de una persona, que corresponde al menos a $10 \%$ por sobre lo recomendado según la talla en el caso de SP y sobre $20 \%$ en OB y producto de un exceso de grasa corporal (1).

La obesidad infantil (OBI) se asocia a un mayor riesgo cardiovascular a edades muy tempranas, que inducen con alta frecuencia un hiperinsulinemia, menor tolerancia a la glucosa, alteraciones en el perfil lipídico en sangre e incluso hipertensión arterial. Los cambios metabólicos observados en niños y adolescentes obesos se conocen también como síndrome pre-metabólico y pueden estar relacionados con los trastornos endocrinos que se observan en la obesidad (2).

En el año 2013 alrededor de 42 millones de niños menores de cinco años tenían malnutrición por exceso $(\mathrm{SP}+\mathrm{OB})$ en el mundo (3). En Chile durante el año 2012, a través del análisis de los "controles de niño sano" de la red pública de salud, por primera vez en Chile la OBI alcanzó una cifra de dos dígitos,
Marcela Ñ unque G. (1)

Lilian Salazar L. (1)

Carina Valenzuela A. (2)

(1) Universidad de Valparaíso Valparaíso, Chile.

(2) Escuela de Nutrición y Dietética. Facultad de Farmacia. Valparaíso, Chile.

Dirigir la correspondencia a: Profesora

Carina Valenzuela A.

Escuela Nutrición y Dietética

Facultad de Farmacia, U niversidad de Valparaíso

Gran Bretaña 1193, Playa Ancha, Valparaíso, Chile

Teléfono: +56 (32) 2508264

Código postal: 2360102

Email: carina.valenzuela@uv.cl

Este trabajo fue recibido el 25 de Agosto de 2014 aceptado con modificaciones el 23 de Julio de 2015 y aceptado para ser publicado el 25 de Agosto de 2015.

con una prevalencia de $10,3 \%$ (4). En cuanto a la prevalencia de SP infantil, aumentó de $32.3 \%(5,6)$ a $32,8 \%(5,6)$, lo que sitúa al exceso de peso infantil como el principal problema de salud pública en este grupo de edad, presentando además una alta carga de morbilidad para las generaciones futuras, y por ende para toda la población.

Estudios indican que un excesivo consumo de alimentos de alta densidad energética, ricos en grasas saturadas, azúcar y sal, y la falta de actividad física representan en la actualidad el estilo de vida de gran parte de los niños chilenos (7- 9). En Chile, casi el $90 \%$ de los adultos, y el $80 \%$ de los adolescentes entre 15 y 19 años son sedentarios (4).

Este desequilibrio entre la ingesta y el gasto energético, sostenido por períodos prolongados de tiempo se reconoce internacionalmente como la principal causa de la elevada y creciente prevalencia de obesidad, lo que ha llevado a organismos internacionales a plantear a los gobiernos la necesidad de prevenir el problema con medidas que promuevan una alimentación saludable y actividad física adecuada en los niños, involucrando especialmente a los sectores de educación, salud, la industria alimentaria y los medios de comunicación, en el marco de una adecuada regulación (9).

Según la "Encuesta Nacional de Calidad de Vida y Salud" 
(10) $49,8 \%$ de las familias encuestadas en la Región M etropolitana refiere comer fuera del hogar al menos 1 vez durante la semana. Al consultar por la cantidad de veces que comen fuera, $19,1 \%$ dice hacerlo de 1 a 3 veces a la semana y $22,3 \%$ de 4 a 5 veces a la semana. Esto representa una gran oportunidad de desarrollo para el rubro de los restoranes, entendidos como los establecimientos destinados a la elaboración y expendio de platos preparados para ser consumidos en el mismo local o para llevar. Los restoranes familiares (RF) se denominan así ya que la decoración y atención tienen un aspecto informal y los restoranes de comida rápida (RCR) se caracterizan por proveer la comida después de pagar.

Recientemente un estudio descriptivo en España, publicado por la revista Eroski Consumer (11), analizó 180 restoranes de 14 ciudades evaluados mediante una tabla calificadora adaptada de la Estrategia para la Nutrición, Actividad Física y Prevención de la Obesidad (NAOS) y Asociación Española de Pediatría (AEP), mostró que $43 \%$ de los menús analizados incluyen platos en los que se han utilizado técnicas culinarias que aportan grasas a los alimentos.

Otro estudio, en Estados Unidos a las 50 mayores cadenas de restoranes, evaluó la calidad nutricional de los menús infantiles mediante una tabla calificadora basada en las guías alimentarias estadounidenses y adaptada por un panel de expertos desde el "National Alliance for N utrition and Activity's M odel Local School Wellness Policies on Physical Activity and Nutrition", arrojó que de los 22 restoranes que tenían menús para niños e información nutricional disponible, 99\% fueron clasificados como de mala calidad nutricional (12).

Un estudio realizado en M éxico, compararon menús infantiles ofrecidos en restoranes convencionales y de comida rápida, concluyendo que ambos menús eran poco saludables tomando como referencia los valores e ingestas recomendadas para el grupo etario establecidos por Food and Nutrition Board (FNB) y el Institute of Medicine of the National Academies (IOM) (13).

Otro aspecto importante de considerar al analizar la oferta alimentaria es su valor económico, ya que se le atribuye un mayor costo a una alimentación saludable, lo que se verificó en un metanálisis realizado por la Escuela de Salud Pública de Harvard (14), donde se encontraron diferencias de 1,5 dólares entre el costo de una alimentación saludable y no saludable.

Este estudio tuvo por objetivo evaluar cuali-cuantitativamente menús infantiles ofrecidos en RF y RCR de la ciudad de Santiago, analizando su calidad nutricional, propiedades saludables y precios.

\section{ATERIALES Y M ÉTODOS}

Se trata de un estudio observacional, descriptivo, cualitativo-cuantitativo de tipo transversal. La muestra estuvo constituida por un total de 45 restoranes ubicados en la ciudad de Santiago; 40 de ellos corresponden a RF y 5 a RCR, los cuales ofrecían dentro de sus menús la opción de "menú infantil" u "opción de plato para niños". La inclusión de los RF fue de acuerdo a nivel socioeconómico (NSE), seleccionando aquellos que estuvieran ubicados en las comunas más representativas de cada NSE, Vitacura y Las Condes para ABC1, Ñ uñoa y Santiago para C2, M aipú para C3, Peñalolén y San Bernardo para D. Se escogieron al azar 10 restoranes por NSE, mediante un listado aleatorio publicado en las páginas amarillas de publiguía según comuna. En el caso de RCR, se incluyeron aquellas cadenas con mayor cantidad de sucursales en el país. En los restoranes que contaban con más de una opción de menú infantil, se eligió la opción más vendida. Se excluyeron en ambos tipos de restoranes aquellos que no poseían resolución sanitaria vigente para el año 2013. Se incluyó arbitrariamente un RCR que no contaba con menú infantil, pero que es el RCR con mayor cantidad de sucursales a lo largo del país.

En ambos tipos de restoranes se compraron los menús infantiles solicitándolos para llevar y se les realizó un análisis cualitativo, evaluando las características de la constitución de los menús, con sus complementos o extras (bebestible, postre, ensalada, aderezos), a partir de una tabla de cumplimiento de criterios de alimentación saludable, en base a las Guías alimentarias para la población chilena aprobadas por el M inisterio de Salud el año 2013 (15). Sin embargo 5 de los mensajes saludables que estas contenían no pudieron ser evaluados por razones metodológicas y fueron incluidos 2 criterios adicionales a la tabla, para evaluar otros aspectos considerados relevantes en la oferta alimentaria. Constituyéndose una tabla con 10 parámetros a evaluar, otorgando un valor nominal de 1 punto por criterio cumplido y 0 punto por criterio no cumplido (tabla 1). Clasificados posteriormente como "muy malo", "malo", "regular", "bueno" o "muy bueno" de acuerdo a la puntuación total obtenida (tabla 2).

Luego se realizó el análisis cuantitativo, estimando la composición nutricional sólo del plato principal de cada menú, pesando cada componente en una balanza analítica Digital Price Computing Scale (con precisión de $5 \mathrm{~g}$ ). Para esta estimación no se consideraron los componentes extras del menú, ya que no todos los menús estaban constituidos de la misma forma.

La estimación del aporte nutricional de cada plato, fue calculado utilizando el peso de cada componente del menú listo para ser consumido y la información nutricional disponible en las Tablas de Composición Química de los Alimentos Chilenos 2004, a excepción de las papas fritas prefritas, donde se utilizó el programa Food Processor ya que los datos presentes en la Tabla de Composición Q uímica de Alimentos Chilena estaban incompletos. Posteriormente se realizó el cálculo de densidad energética $(D E)$, porcentaje de proteínas $(P \%)$, hidratos de carbono $(\mathrm{CHO} \%)$ y grasas $(\mathrm{G} \%)$ del valor calórico total y la proporción de aporte de ácidos grasos (relación saturados: monoinsaturados: poliinsaturados) y aporte de sodio (mg).

La comparación de aporte de energía (kcal), macronutrientes y sodio, se realizó con las recomendaciones nutricionales correspondientes para el tiempo de comida de almuerzo (equivalente al $30 \%$ del valor calórico total VCT) para dos grupos etarios (16), preescolar de 2 a 5 años y escolar de 6 a 9 años, considerando una actividad física moderada para ambos grupos (tablas 3 y 4 ).

Para el análisis estadístico de los datos se utilizó el programa Statgraphic Centurion Versión XVI, utilizando pruebas de correlación y T-Student para resultados cuantitativos y la prueba Chi Cuadrado, para los resultados cualitativos. Las variables estudiadas fueron precios, recomendaciones nutricionales, aporte energético y nutricional de los menús, calidad nutricional, características saludables de acuerdo al cumplimiento de los tópicos de las nuevas guías alimentarias, para ambos restoranes. Se consideró una significancia estadística con un valor predictivo menor a 0,05 $(p<0,05)$.

No existió conflicto de interés de los autores de este trabajo, con los restoranes que formaron parte de este estudio.

\section{RESULTADOS}

La tabla 5 presenta los aportes y adecuaciones de energía, macronutrientes y sodio de RF y RCR de los menús estudiados, y se observa que ambos tipos de restoranes sobrepasan la ma- 
yoría de las recomendaciones nutricionales correspondientes al almuerzo, principalmente para el grupo de preescolares, siendo la energía, proteínas, lípidos y sodio los parámetros que mayormente excedían las recomendaciones para este grupo. Sorpresivamente, los aportes nutricionales de los menús de los RF excedían en mayor medida a las recomendaciones nutricionales al compararlos con los RCR, a excepción del sodio. En energía, los RF aportan $84 \%$ extra por sobre las recomendaciones, mientras que sobrepasan $170 \%$ en el caso de proteínas y $153 \%$ en los lípidos. En los escolares, al presentar requerimientos nutricionales mayores, estos parámetros se exceden en menor magnitud. El sodio es el parámetro que mayormente sobrepasaba las recomendaciones, con una adecuación de $328 \%$ en el caso de los RF y $742 \%$ en los RCR.

Al realizar la correlación entre el precio y la proporción de macronutrientes de ambos restoranes son las proteínas las únicas que muestran una relación directa de forma significativa, es decir, a mayor precio mayor proporción de proteínas en el menú como se observa en la figura 1. M ientras que los hidratos de carbono muestran una correlación negativa y las grasas presentan una correlación positiva con el precio de los menús, sin embargo estas no presentan significancia estadística.

En cuanto a las características saludables, la tabla 6 muestra el porcentaje de cumplimiento de cada criterio evaluado en los

TABLA 1

Criterios de calidad saludable en base a las Guías Alimentarias 2013 para clasificar los menús infantiles.

\begin{tabular}{|c|c|c|c|}
\hline $\begin{array}{l}\text { M ensajes saludables } \\
\text { seleccionado }\end{array}$ & Parámetro a evaluar & Puntaje & Bases científicas \\
\hline $\begin{array}{c}\text { "Come alimentos con poca } \\
\text { sal y saca el salero de la } \\
\text { mesa" }\end{array}$ & $\begin{array}{l}\text { El plato principal está constituido en } \\
\text { menos de un } 50 \% \text { por productos pre } \\
\text { elaborados como "embutidos" y/o } \\
\text { "productos pre fritos". }\end{array}$ & 1 & $\begin{array}{l}\text { La ingesta de sodio afecta los niveles de pre- } \\
\text { sión arterial a nivel poblacional. El } 62 \% \text { de las } \\
\text { enfermedades cerebro vasculares y } 49 \% \text { de las } \\
\text { cardiopatías isquémicas (20) se debieron a la } \\
\text { elevación de la presión arterial. }\end{array}$ \\
\hline $\begin{array}{l}\text { "Cuida tu corazón evitan- } \\
\text { do las frituras y alimentos } \\
\text { con grasas como cecinas y } \\
\text { mayonesa" }\end{array}$ & $\begin{array}{l}\text { El plato principal está constituido en } \\
\text { menos de un } 50 \% \text { por alimentos con } \\
\text { técnicas culinarias poco grasas como } \\
\text { "al vapor", "a la plancha" o "cocido } \\
\text { en agua". }\end{array}$ & 1 & $\begin{array}{l}\text { La ingesta de grasas no debe superar } 25-30 \% \\
\text { del VCT, siendo la proporción de AGS, AGM I } \\
\text { y AGPI de } 1: 1: 1(16) \text {. }\end{array}$ \\
\hline $\begin{array}{l}\text { "Come } 5 \text { veces verduras y } \\
\text { frutas frescas de distintos } \\
\text { colores, cada día" }\end{array}$ & $\begin{array}{c}\text { Se ofrecen verduras como entrada o } \\
\text { ensalada. } \\
\text { Se ofrece fruta fresca entera o picada } \\
\text { como postre. } \\
\text { Ofrece jugo natural o de pulpa como } \\
\text { bebestible. }\end{array}$ & $\begin{array}{l}1 \\
1 \\
1\end{array}$ & $\begin{array}{l}\text { El consumo insuficiente de frutas y verduras } \\
\text { es uno de los riesgos para desarrollar ECNT, } \\
\text { ya que aporta vitaminas, minerales y fitoquí- } \\
\text { micos }(20) \text {. }\end{array}$ \\
\hline $\begin{array}{c}\text { “Para mantener sano tu } \\
\text { corazón, come pescado al } \\
\text { horno o a la plancha, } 2 \text { veces } \\
\text { por semana" }\end{array}$ & $\begin{array}{c}\text { El plato principal ofrece pescado o } \\
\text { productos marinos. }\end{array}$ & 1 & $\begin{array}{l}\text { Los productos del mar tienen un alto contenido } \\
\text { de omega } 3 \text {, el cual interviene positivamente } \\
\text { en los procesos pro-inflamatorios, anticoagu- } \\
\text { lantes, disminución de los niveles de colesterol } \\
\text { y triglicéridos, además promueve el control de } \\
\text { la presión sanguínea (16). }\end{array}$ \\
\hline $\begin{array}{l}\text { "Consume legumbres al me- } \\
\text { nos dos veces por semana, } \\
\text { sin mezclarlas con cecinas" }\end{array}$ & $\begin{array}{c}\text { El plato principal, o ensalada contiene } \\
\text { legumbres. }\end{array}$ & 1 & $\begin{array}{l}\text { Legumbres: contienen antioxidantes y fibra } \\
\text { que ayudan a prevenir las enfermedades car- } \\
\text { diovasculares y algunos tipos de cáncer (16). }\end{array}$ \\
\hline $\begin{array}{l}\text { "Lee y compara las etiquetas } \\
\text { de los alimentos y prefiere } \\
\text { los que tengan menos grasas, } \\
\text { azúcar y sal (sodio)" }\end{array}$ & $\begin{array}{l}\text { Los menús cuentan con información } \\
\text { nutricional. }\end{array}$ & 1 & $\begin{array}{l}\text { Con el fin de preferir alimentos bajos en } \\
\text { calorías, azúcar, grasas saturadas, entre otras } \\
(13,16) \text {. }\end{array}$ \\
\hline Incluido & $\begin{array}{l}\text { Se ofrece menús especiales sin sal, sin } \\
\text { gluten y/o vegetariano. }\end{array}$ & 1 & $\begin{array}{l}\text { Basado en estudio realizado en Eroski Cosu- } \\
\text { mer (11). }\end{array}$ \\
\hline Incluido & $\begin{array}{c}\text { Se ofrece aceite de oliva como ade- } \\
\text { rezo. }\end{array}$ & 1 & $\begin{array}{l}\text { Aceite de oliva: Debe preferirse este tipo de } \\
\text { grasas ya que reduce LDL aumenta HDL. (16). }\end{array}$ \\
\hline
\end{tabular}

TABLA 2

Clasificación de los menús infantiles según puntaje final obtenido en los criterios de calidad saludable.

\begin{tabular}{lccccc}
\hline Clasificación & “M uy mala” & “M ala” & “Regular” & “Buena” & “M uy buena” \\
\hline Puntos obtenidos & $0-2$ & $3-4$ & $5-6$ & $7-8$ & $9-10$ \\
\hline
\end{tabular}


RF, RCR y en el total de la muestra. Se observó que ningún menú estudiado cumple en $100 \%$ los criterios de una alimentación saludable, ya que ninguna muestra obtuvo el puntaje máximo de 10 puntos, y un total de 6 muestras obtuvieron un puntaje 0 . Al comparar ambos tipos de restoranes, observamos que existe un menor cumplimiento de criterios por parte de los restoranes de RCR, obteniendo $0 \%$ de cumplimiento en 7 de los 10 criterios y donde su mayor aprobación fue de $40 \%$ en la oferta de fruta

FIGURA 1

Correlación entre el precio y aporte de proteínas (g)

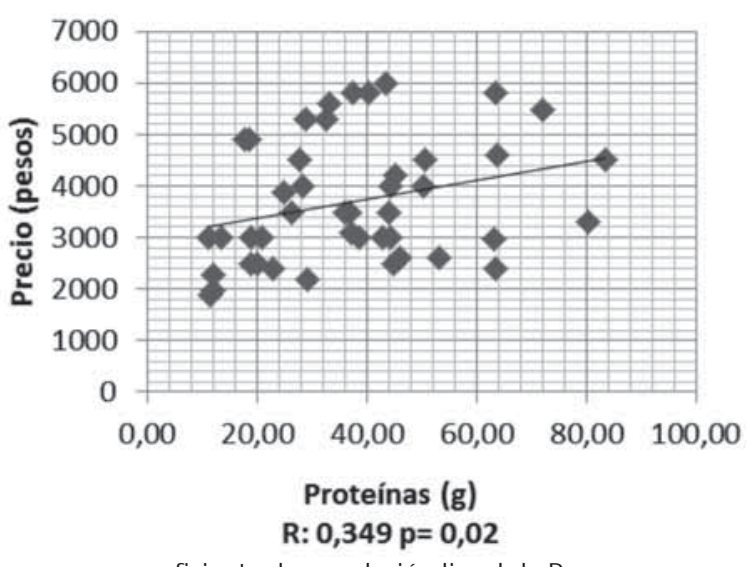

r: coeficiente de correlación lineal de Pearson.

TABLA 3

Recomendaciones de energía y macronutrientes diaria y para el tiempo de comida de almuerzo.

\begin{tabular}{|c|c|c|c|c|c|c|}
\hline & \multicolumn{3}{|c|}{ Preescolar } & \multicolumn{3}{|c|}{ Escolar } \\
\hline Edad & \multicolumn{3}{|c|}{2 a 5 años } & \multicolumn{3}{|c|}{6 a 9 años } \\
\hline Recomendación de energía & $\begin{array}{l}\text { Hombres } \\
1400 \mathrm{kcal} / \mathrm{d}\end{array}$ & & $\begin{array}{c}\text { M ujeres } \\
1250 \mathrm{kcal} / \mathrm{d}\end{array}$ & $\begin{array}{l}\text { Hombres } \\
1700 \mathrm{kcal} / \mathrm{d}\end{array}$ & & $\begin{array}{c}\text { M ujeres } \\
1550 \mathrm{kcal} / \mathrm{d}\end{array}$ \\
\hline $\begin{array}{c}\text { Promedio de recomendación } \\
\text { de energía }\end{array}$ & \multicolumn{3}{|c|}{$1325 \mathrm{kcal} / \mathrm{d}$} & \multicolumn{3}{|c|}{$1625 \mathrm{kcal} / \mathrm{d}$} \\
\hline $\begin{array}{l}\text { Recomendación (rec) de } \\
\text { energía al almuerzo ( } 30 \% \text { del } \\
\text { aporte diario) }\end{array}$ & \multicolumn{3}{|c|}{$400 \mathrm{kcal}$} & \multicolumn{3}{|c|}{$490 \mathrm{kcal}$} \\
\hline $\begin{array}{c}\text { Proteínas } \\
\text { (12 - } 15 \% \text { del VCT) }\end{array}$ & $\begin{array}{l}\text { Rango aporte } \\
\text { diario (gr/día) } \\
40-50 \\
\end{array}$ & $\begin{array}{c}\bar{x} \\
45 \mathrm{~g} \\
\end{array}$ & $\begin{array}{l}\text { Rec. al } \\
\text { almuerzo } \\
14 \mathrm{~g}\end{array}$ & $\begin{array}{c}\text { Rango aporte } \\
\text { diario (gr/día) } \\
49-61 \mathrm{~g} \\
\end{array}$ & $\begin{array}{c}\bar{x} \\
55 \mathrm{~g} \\
\end{array}$ & $\begin{array}{l}\text { Rec. al } \\
\text { almuerzo } \\
17 \mathrm{~g} \\
\end{array}$ \\
\hline $\begin{array}{c}\text { Lípidos } \\
\text { (25-30\% del VCT) }\end{array}$ & $37-52$ & $45 \mathrm{~g}$ & $14 \mathrm{~g}$ & $45-63 g$ & $54 \mathrm{~g}$ & $16 \mathrm{~g}$ \\
\hline $\begin{array}{l}\text { Hidratos de carbono } \\
\text { (50-60\% del VCT) }\end{array}$ & $166-199$ & $183 \mathrm{~g}$ & $55 \mathrm{~g}$ & $203-244 \mathrm{~g}$ & $244 \mathrm{~g}$ & $73 \mathrm{~g}$ \\
\hline
\end{tabular}

\% del VCT: porcentaje del valor calórico total.

TABLA 4

Recomendación de sodio por día y en el almuerzo (30\%) según edad en niños (IOM N, NASs, USA, 2005).

\begin{tabular}{cccc}
\hline Edad & Sodio/día & $30 \%$ de la recomendación diaria & $\begin{array}{c}\text { Recomendación promedio } \\
\text { de sodio en el almuerzo }\end{array}$ \\
\hline $1-3$ años & $1000 \mathrm{mg}$ & $300 \mathrm{mg}$ & $330 \mathrm{mg}^{*}$ \\
$4-8$ años & $1200 \mathrm{mg}$ & $360 \mathrm{mg}$ &
\end{tabular}

*Promedio de las recomendaciones de los datos para niños de 1-3 años y 4-8 años que entrega la IOM N, NASs, USA, 2005. 
como postre. Los RF cuentan con mayor puntaje de aprobación en los criterios evaluados, sin embargo, 8 de los 10 criterios son cumplidos por menos de $50 \%$ de los menús estudiados.

Para el análisis de asociación entre las variables precio y cumplimiento de Guías Alimentarias, se aplicó la prueba Chi cuadrado que muestra una asociación positiva entre las variables, sin embargo esta asociación no fue significativa $(p=0,129)$, por lo que el cumplimiento de las guías alimentarias no depende del precio del menú.

\section{DISCUSIÓN}

Los resultados muestran que tanto los menús infantiles ofrecidos en RF como en RCR sobrepasan las recomendaciones nutricionales de energía, macronutrientes y sodio para preescolares y escolares para el almuerzo, siendo los RF quienes sobrepasan en mayor magnitud dichos parámetros. Se observó que los menús infantiles ofrecidos en ambos tipos de restoranes son deficientes en calidad nutricional y que existe una tendencia a una asociación positiva entre el precio del menú y la densidad energética, aporte de grasas y proteínas, siendo sólo esta última la que presentó una significancia estadística.
No existen estudios chilenos actuales que hagan referencia a esta área de investigación lo cual impide comparar nuestros resultados con otros datos nacionales. Investigaciones realizadas en otros países obtuvieron resultados similares a los nuestros, como un exceso de aporte energético, de hidratos de carbono, grasas y proteínas de los menús. En un estudio realizado en M éxico (13) compararon los menús infantiles de cadenas de comida rápida y de restoranes convencionales y encontraron que ambos sobrepasaban las recomendaciones nutricionales infantiles.

Respecto a las diferencias de aporte energético y macronutrientes encontrados al comparar los menús de ambos tipos de restoranes, los RF aportaban aproximadamente $260 \mathrm{kcal}$ y 22 gramos de proteínas más que los de RCR. Esta situación se contrasta con lo reportado en el estudio antes mencionado, en el cual eran los RCR quienes presentaban aportes más altos. Esta diferencia se podría deber a que en los RF las porciones entregadas eran similares a las que se ofrecen a los adultos, existiendo menús infantiles que contenían hasta $410 \mathrm{~g}$ de papas fritas por plato principal, lo que se observó en menor medida en los locales de comida rápida, donde existe una estandarización

TABLA 5

Aportes y adecuaciones de energía, macronutrientes y sodio de menús infantiles según tipo de restoran.

\begin{tabular}{|c|c|c|c|c|c|c|c|}
\hline \multicolumn{2}{|c|}{$\begin{array}{l}\text { NUTRIENTES } \\
\text { Promedio } \pm \text { Desviación } \\
\text { Estándar }\end{array}$} & $\begin{array}{l}\text { Energía (kcal) } \\
\qquad \bar{x} \pm \mathrm{DE}\end{array}$ & $\begin{array}{l}\text { Proteínas (g) } \\
\qquad \bar{x} \pm \mathrm{DE}\end{array}$ & $\begin{array}{l}\mathrm{CHO}(\mathrm{g}) \\
\bar{x} \pm \mathrm{DE}\end{array}$ & $\begin{array}{l}\text { Lípidos (g) } \\
\bar{x} \pm \mathrm{DE}\end{array}$ & \multicolumn{2}{|c|}{$\begin{array}{c}\text { Sodio (mg) } \\
\quad \pm D E\end{array}$} \\
\hline & RF & $735 \pm 292$ & $37,8 \pm 18,5^{*}$ & $68,1 \pm 28$ & $35,3 \pm 21,7$ & \multicolumn{2}{|c|}{$1084,2 \pm 516,9$} \\
\hline \multirow[t]{3}{*}{ Aporte } & $\mathrm{RCR}$ & $514 \pm 205$ & $17,1 \pm 8,5^{*}$ & $53,1 \pm 32,2$ & $24,2 \pm 5,9$ & \multicolumn{2}{|c|}{$989,6 \pm 387,8$} \\
\hline & \multicolumn{7}{|l|}{ Total } \\
\hline & muestra & $710 \pm 290$ & $35,4 \pm 18,8$ & $66,4 \pm 28,5$ & $34,2 \pm 20,7$ & \multicolumn{2}{|c|}{$1073,4 \pm 542,2$} \\
\hline \multicolumn{2}{|c|}{$\begin{array}{l}\text { Adecuación según lo } \\
\text { recomendado para el almuerzo }\end{array}$} & $\%$ & $\%$ & $\%$ & $\%$ & \multicolumn{2}{|c|}{$\%$} \\
\hline \multirow[t]{2}{*}{ Preescolares } & $\mathrm{RF}$ & 184 & 270 & 124 & 253 & RF & $\mathrm{RCR}$ \\
\hline & $\mathrm{RCR}$ & 129 & 122 & 97 & 173 & \multirow{3}{*}{328} & \multirow{3}{*}{742} \\
\hline \multirow[t]{2}{*}{ Escolares } & RF & 150 & 222 & 93 & 221 & & \\
\hline & RCR & 105 & 101 & 72 & 151 & & \\
\hline
\end{tabular}

DE: Desviación estándar; RF: Restoranes familiares; RCR: Comida rápida.; t-student $*^{*}(<0.018)$

TABLA 6

Porcentajes de cumplimiento de los criterios de calidad saludable según tipo de restoran.

\begin{tabular}{|c|c|c|c|}
\hline Criterios de calidad saludable & RF & $\mathrm{RCR}$ & Total* \\
\hline \multicolumn{4}{|l|}{ El plato principal está constituido en menos de un $50 \%$ por productos } \\
\hline $\begin{array}{l}\text { pre elaborados como "embutidos" y/o "productos pre fritos" } \\
\text { El plato principal está constituido en menos de un } 50 \% \text { por alimentos }\end{array}$ & $48 \%$ & $0 \%$ & $42 \%$ \\
\hline \multicolumn{4}{|l|}{ con técnicas culinarias poco grasas como "al vapor", "a la plancha" o } \\
\hline "cocido en agua". & $43 \%$ & $0 \%$ & $38 \%$ \\
\hline Se ofrecen verduras como entrada o ensalada. & $40 \%$ & $20 \%$ & $38 \%$ \\
\hline Se ofrece fruta fresca entera o picada como postre. & $18 \%$ & $40 \%$ & $20 \%$ \\
\hline Se ofrece jugo de fruta natural o de pulpa como bebestible. & $58 \%$ & $0 \%$ & $51 \%$ \\
\hline El plato principal ofrece pescado o productos marinos. & $25 \%$ & $0 \%$ & $22 \%$ \\
\hline El plato principal, o ensalada contiene legumbres. & $3 \%$ & $0 \%$ & $2 \%$ \\
\hline Los menús cuentan con información nutricional. & $0 \%$ & $20 \%$ & $2 \%$ \\
\hline Se ofrece menús especiales sin sal, sin gluten y/o vegetariano. & $28 \%$ & $0 \%$ & $24 \%$ \\
\hline Se ofrece aceite de oliva como aderezo. & $60 \%$ & $0 \%$ & $53 \%$ \\
\hline
\end{tabular}

RF: Restoranes Familiares; RCR: Comida Rápida.

*Total: \% del total de los restoranes, sumando los RF y RCR, que cumplieron con el criterio respetivo. 
del tamaño de las porciones. Ejemplo de esto es que 12 menús del total superan las $800 \mathrm{kcal}$, las cuales representan la mitad de las recomendaciones de energía de un niño al día (pre-escolar o escolar), mientras que 34 muestras superan el $30 \%$ del valor calórico total de grasas (25-30\% recomendación).

Esta ingesta aumentada de energía puede ser una de las variables contribuyentes a agudizar el panorama epidemiológico nacional, donde la malnutrición por exceso es el principal problema de salud pública en este grupo etario, dejando a Chile como el país con mayor obesidad en menores de 5 años a nivel Latinoamericano (17).

Está comprobado que el exceso de consumo alimentario sostenido desde la niñez en adelante propicia una carga de morbilidad importante en la vida adulta y se ha descrito que las ECNT (enfermedades crónicas no transmisibles) están apareciendo cada vez más precozmente, por lo que se podría estimar que en los próximos años esta situación se agrave.

Si proyectamos que un niño con estado nutricional normal comiera fuera de casa 1 vez a la semana, al cabo de 1 mes consumiría en promedio $1.060 \mathrm{kcal}, 76,5 \mathrm{~g}$ proteínas, 77,6 $\mathrm{g}$ lípidos y 69,6 g de hidratos de carbono adicionales a sus requerimientos, esto si consideramos que el resto de lo que consume en el día es adecuado.

Si esta proyección se sostiene a lo largo de 1 año, el mismo niño consumiría 12.720 kcal extras obtenidos sólo con el consumo del almuerzo fuera del hogar, independiente si lo hace en un restoran de comida rápida o convencional, significando aproximadamente la ganancia de $1,8 \mathrm{Kg}$ de grasa extra. Este aumento de peso corporal en un niño puede definir si presenta SP u OB. Por esta razón, se deben planificar nuevas estrategias para abordar la OBI de una forma integral, diseñando intervenciones que se adapten al estilo de vida actual, como también apoyando la producción, comercialización y publicidad de alimentos saludables (18), para así generar una competencia más equitativa frente a los alimentos no saludables.

En relación a la evaluación de la calidad nutricional de la oferta alimentaria para niños, existen pocas investigaciones que lo hayan considerado como parámetro en el análisis de menús infantiles. Al analizar las características saludables de los menús, tomando como referencia el cumplimiento de los mensajes saludables de las Guías Alimentarias Chilenas del año 2013, a través de las cualidades de la composición de cada menú, fue posible observar que existe un mayor cumplimiento de estos criterios por parte de los RF, con sólo 1 criterio no observado. En cambio los menús de RCR poseen incumplimiento total en 7 de los 10 criterios utilizados. Alrededor del $80 \%$ de la totalidad de los menús fueron clasificados como "malo" o "muy malo", es decir, cumplieron con menos de 4 criterios. Los parámetros menos cumplidos fueron: "el plato principal, o ensalada contiene legumbres" y "Ios menús cuentan con información nutricional". M ientras que los parámetros con mayor puntaje fueron "ofrece aceite de oliva como aderezo" y "se ofrece jugo natural o de pulpa como bebestible". El 100\% de los restoranes de RCR y alrededor del 50\% de los RF utilizan productos embutidos, pre-fritos y técnicas culinarias grasas en la preparación de su menú, y en promedio $40 \%$ de estos ofrece verduras como ensaladas o como parte del plato principal. Estos resultados se asemejan a los obtenidos por la revista Eroski Consumer el año 2012 (11), en el que analizaron 180 restoranes de diferentes ciudades de España, donde $43 \%$ de los menús analizados incluían platos con técnicas culinarias que aportaban grasas a los alimentos y $22 \%$ incluyó como mínimo un plato de verduras o ensaladas en su oferta. En el criterio que hace referencia a la utilización de fruta, se observa que en más del $60 \%$ de ambos tipos de restoranes, no se utilizan las frutas como opción de postre. En $40 \%$ de los restoranes de RCR se reemplazó el helado por manzana conservada (deshidratada o envasada). Adicionalmente, se observó una inclusión casi nula de pescado o productos marinos como parte del plato principal, independiente del tipo de restoran; lo mismo ocurre con las legumbres.

Un antecedente importante a destacar es que ningún RF cuenta con información nutricional de los platos que se ofrecen para los niños. Sólo un restorán del total de la muestra contaba con información nutricional, el cual corresponde a un RCR internacional. Con respecto a la existencia de menús especiales como vegetariano, sin gluten o sin sal dentro de la oferta para niños, se observó que alrededor de $30 \%$ de los RF y $0 \%$ de los RCR cuentan con esta opción. Sin embargo, esto ocurre sólo al solicitar un menú bajo en sal, ya que ningún restoran de la muestra contaba con una opción establecida para el resto de menús especiales.

El promedio de los precios fue $\$ 3.711$ pesos, siendo $\$ 6.000$ pesos el mayor valor cancelado en un RF y $\$ 1.890$ pesos (de RCR) el menor. Un aspecto a considerar en este mismo ámbito, es que los menús infantiles ofrecidos en restoranes de RCR presentaron menores precios que los RF, Io que se podría atribuir a la gran competencia entre los RCR al estar ubicados por lo general en patios de comida, siendo el principal objetivo de cada local incentivar la elección de sus menús en base a la mejor oferta.

En relación a las debilidades del estudio, hubiese sido óptimo realizar un análisis proximal de los menús infantiles analizados para obtener la composición nutricional exacta de estos, es decir su aporte energético, de macronutrientes, micronutrientes y de humedad (valor necesario para poder estimar de forma exacta la densidad energética de los alimentos). Sin embargo, esta técnica analítica posee un costo elevado por lo que no fue posible utilizarla. Pese a esto, la técnica utilizada tomó como parámetro de referencia la Tabla de Composición Química de Alimentos Chilena 2004 (19), creada a partir de análisis proximal, y que es utilizada por la mayoría de los profesionales para estimar aportes energéticos y de macronutrientes. Consideramos necesaria la realización de nuevos estudios que utilicen mejores técnicas de estimación de aporte nutritivo y que abarquen mayor cantidad de muestras, así como también otras regiones del país. Adicionalmente, nuevos estudios requieren del diseño de instrumentos más idóneos para evaluar los parámetros saludables de la oferta alimentaria infantil de restoranes.

Esta investigación, además de obtener un diagnóstico sobre la oferta alimentaria infantil local, tuvo como propósito contribuir en la entrega de antecedentes que impulsen una mayor regulación de la industria alimentaria, al existir escasos estudios que abordan este tópico a nivel nacional y mundial.

Para que se lleven a cabo cambios relevantes en relación a la oferta alimentaria dirigida a los niños consideramos necesario el trabajo conjunto de la industria gastronómica, asociaciones de consumidores, las familias y el gobierno. Este último quien debe velar para que la industria cumpla las leyes y reglamentos vigentes, además de guiarse por los lineamientos de la OMS que el año 2010 instó limitar la cantidad de publicidad de alimentos dirigidos a niños. Países de todo el mundo trabajan para combatir la obesidad y los factores que la predisponen, sobre todo en niños, mediante legislaciones que apuntan a controlar el marketing, creando mensajes nutricionales positivos o de advertencia, utilizando esta forma 
para educar a la población sobre alimentación saludable e incentivando una mejor elección del alimento. Es por esto que en Chile según la Ley 22.606, "sobre composición nutricional de los alimentos y su publicidad" (Promulgada el 6/6/12) se prohíbe la publicidad de alimentos catalogados por el M INSAL como "altos en grasa", "altos en sodio" (Art. 5, Art 8).

En conclusión, en la muestra estudiada se observó que la oferta de menús infantiles en Chile excede el aporte nutricional recomendado para los niños en el almuerzo y que tienen características poco saludables, ya sea por la cantidad de alimentos, tipo de preparaciones y su calidad nutricional. Se estima desde esta perspectiva, que el comer frecuentemente fuera del hogar, en RF o en RCR, podría contribuir al desarrollo de malnutrición por exceso en la infancia. Para contrarrestar esta situación, a nivel de salud pública se deberán priorizar intervenciones de carácter preventivo y de promoción de la salud, que impliquen educar a la población y entregarles herramientas que les permitan tomar decisiones informadas. Si a esto le sumamos una oferta de alimentos más variada, mayor disponibilidad de alimentos saludables, una regulación firme de publicidad/marketing de alimentos y un mejor etiquetado, sin duda podríamos observar efectos positivos en la salud y calidad de vida de la población.

Agradecimientos: Financiamiento: Este trabajo fue financiado por la organización de consumidores, ODECU.

\section{RESUM EN}

En Chile, la obesidad infantil está en su nivel más alto, el consumo de alimentos fuera del hogar es cada vez más frecuente, siendo la oferta alimentaria infantil cuestionada a nivel mundial por su relación con la obesidad infantil y por poseer características poco saludables. Se evaluaron 45 menús infantiles de restoranes familiares y de comida rápida realizando un análisis cualitativo-cuantitativo. Se observó que la mayoría de los menús estudiados exceden las recomendaciones de grasa y sodio (sobre $150 \%$ y $328 \%$, respectivamente); y $40 \%$ de ellos superaban las 800 calorías, considerada la mitad de las recomendaciones de energía para un niño al día. Así, el aporte de energía entregado por los menús analizados superaba los requerimientos infantiles, no existiendo una relación directa entre el precio y calidad nutricional. Estos menús tienen pocas propiedades saludables, clasificándose $80 \%$ como malos o muy malos según las guías alimentarias para la población chilena publicadas por el MINSAL el año 2013.

Palabras clave: Obesidad infantil, menús infantiles, comida rápida, restoranes familiares, regulación alimentaria.

\section{BIBLIOGRAFIA}

1. Biblioteca nacional de medicina de estados unidos. Disponible en: http://www.nlm.nih.gov/medlineplus/ spanish/ency/article/003101.htm. [Consultado el 22 de mayo de 2013].

2. Dietz WH. Childhood weight affects adult morbidity and mortality. J Nutr. 1998;128:411-414.

3. Organización M undial de la Salud. Disponible en: http:// www.who.int/mediacentre/factsheets/fs311/es. [Consultado el 22 de mayo de 2013].

4. Encuesta Nacional de Salud 2010. M inisterio de Salud. Gobierno de Chile. Disponible en: http://www.redsalud. gov.cl/portal/url/item/9ac1bfled8ee8922e0400101 1e013229.pdf. [Consultado el 10 de mayo de 2013].

5. Análisis controles niño sano, población en control en red de salud pública. M inisterio de Educación Chile. Universidad de Chile. Disponible en: http://radio.uchile. cl/noticias/217627/. [Consultado el 28 de junio de 2013].

6. Situación nacional de población bajo control en el sistema público de salud (menores de 6 años). Encuesta Nacional de Salud 2010. M inisterio de Salud. Gobierno de Chile. Disponible en: http://www.redsalud.gov.cl/portal/url/it em/9aclbfled8ee8922e04001011e013229.pdf. [Consultado el 20 de junio de 2013].

7. Uauy R, Albala C, Kain J. Obesity trends in Latin America: transiting from under to overweight. J Nutr. 2001; 131:8935-95.

8. WHO. World Health Organization Expert Committee on Physical Status. The use and interpretation of anthropometry. Geneva: Report of a WHO Expert Committee; WHO Technical Report Series No 854. 1995.

9. Salinas J, Vio F. Política de promoción de salud en Chile. En: Albala C, Olivares S, Salinas J, Vio F eds. Bases, prioridades y desafíos de la promoción de la salud. Santiago: M inisterio de Salud/Vida Chile/Universidad de Chile/ INTA, Andros Impresores; 2004.

10. Encuesta Nacional de vida y salud 2000. Departamento de epidemiología y departamento de promoción en salud. Instituto Nacional de Estadísticas. Gobierno de Chile. Disponible en: http://epi.minsal.cl/epi/html/sdesalud/ calidaddevida2006/Informe\%20Final\%20Encuesta\%20 de $\% 20$ Calidad\%20de\%20Vida\%20y\%20Salud\%202006. pdf. [Consultado el 6 de junio de 2013].

11. Cuevas R., 2013. M enús infantiles en restaurantes: Poca variedad y baja calidad dietética. Eroski Consumer, 172: 22-27. Disponible en: http://revista.consumer.es/web/ es/20130201/pdf/revista_entera.pdf [Consultado el 10 de marzo de 2013].

12. Batada $A$, Bruening $M, M$ archlewicz $E H$, Historia $M$, Wootan MG. Poor nutrition on the menu: children's meals at America's top chain restaurants. Obes niños. 2012; 8 (3):251-4.

13. Ramos E., Castro-Sánchez A., Zambrano A., Nuñez G., Osorio S. Aporte calórico y macronutrimental de los menús infantiles de la comida rápida y convencional. Rev Chil Nutr. 2012; 39 (3): 27-33.

14. Pulos E., Leng K. Evaluation of a voluntary M enu-labeling program in full-service restaurants. Am J Public Health 2010; 100 (6): 1035-9.

15. Instituto de Nutrición y Tecnología de los Alimentos (INTA). Universidad de Chile. Guías Alimentarias 2013. Disponible en: http://www.inta.cl/doc_noticias/GUIAS_ALIM ENTARIAS_2013.pdf [Consultado el1 5 de junio de 2013].

16. Guía de alimentación del menor de 2 años hasta la adolescencia. Departamento de Nutrición y Ciclo Vital, División de prevención y control de enfermedades. M INSAL 2005.

17. Amigo H. Obesidad en el Niño en Latinoamérica: Situación, criterios de diagnóstico y desafíos. Cad Saude Pública, Rio de Janeiro 2003; 19 (Suppl.1): S163-S70.

18. Castillo C., Romo M. Las Golosinas en la Alimentación Infantil. Rev Chil Pediatr. 2006; 77 (2); 189-93.

19. FAO. Producción y manejo de datos de composición química de alimentos en nutrición: Uso de tablas de composición de alimentos en las intervenciones alimentarias y nutricionales.; Araya H. 1997. p 9-18.

20. Zacarías I., Pizarro T., Rodríguez L., González D., Domper A. Programa "5 al día" para promover el consumo de verduras y frutas en Chile. Rev Chil Nutr. 2006; 33 (Suppl. 1): S276-S80. 